\title{
Three Large-Scale Functional Brain Networks from Resting-State Functional MRI in Subjects with Different Levels of Cognitive Impairment
}

\author{
Soo Hyun Joo', Hyun Kook Lim², and Chang Uk Lee ${ }^{1 凶}$ \\ ${ }^{1}$ Department of Psychiatry, Seoul St. Mary's Hospital, College of Medicine, The Catholic University of Korea, Seoul, Republic of Korea \\ 2Department of Psychiatry, St. Vincent's Hospital, College of Medicine, The Catholic University of Korea, Suwon, Republic of Korea
}

\begin{abstract}
Normal aging and to a greater degree degenerative brain diseases such as Alzheimer's disease (AD), cause changes in the brain's structure and function. Degenerative changes in brain structure and decline in its function are associated with declines in cognitive ability. Early detection of $\mathrm{AD}$ is a key priority in dementia services and research. However, depending on the disease progression, neurodegenerative manifestations, such as cerebral atrophy, are detected late in course of AD. Functional changes in the brain may be an indirect indicator of trans-synaptic activity and they usually appear prior to structural changes in $\mathrm{AD}$. Resting-state functional magnetic resonance imaging (RS-fMRI) has recently been highlighted as a new technique for interrogating intrinsic functional connectivity networks. Among the majority of RS-fMRI studies, the default mode network (DMN), salience network (SN), and central executive network (CEN) gained particular focus because alterations to their functional connectivity were observed in subjects who had $\mathrm{AD}$, who had mild cognitive impairment (MCI), or who were at high risk for AD. Herein, we present a review of the current research on changes in functional connectivity, as measured by RS-fMRI. We focus on the DMN, SN, and CEN to describe RS-fMRI results from three groups: normal healthy aging, $\mathrm{MCI}$ and $\mathrm{AD}$.

Psychiatry Investig 2016;13(1):1-7
\end{abstract}

Key Words Alzheimer's, Aging, Functional connectivity, fMRI, Resting state.

\section{INTRODUCTION}

Worldwide, the elderly population is growing, increasing the concern over age-related cognitive decline. Cognitive decline has a negative impact on quality of life and, healthy brain aging is an essential component of overall well-being. Because of the nearly global rise in life expectancy and the concomitant emphasis on quality of life, healthy brain aging has become an important focus. ${ }^{1}$

Normal aging and to a greater degree degenerative brain diseases such as Alzheimer's disease (AD), cause changes in the brain's structure and function. Degenerative changes in brain structure and decline in its function are associated with

Received: September 8, 2015 Revised: October 19, 2015

Accepted: October 19, 2015 Available online: November 20, 2015

$\triangle$ Correspondence: Chang Uk Lee, MD, PhD

Department of Psychiatry, Seoul St. Mary's Hospital, College of Medicine, The Catholic University of Korea, 222 Banpo-dearo, Seocho-gu, Seoul 06591, Republic of Korea

Tel: +82-2-2258-6082, Fax: +82-2-594-3870, E-mail: jihan@catholic.ac.kr

(a) This is an Open Access article distributed under the terms of the Creative Commons Attribution Non-Commercial License (http://creativecommons.org/licenses/by$\mathrm{nc} / 3.0$ ) which permits unrestricted non-commercial use, distribution, and reproduction in any medium, provided the original work is properly cited. declines in cognitive ability. Mild-to-moderate age-dependent brain volume loss, enlarged periventricular (VirchowRobin) spaces, punctiform or minor white matter abnormalities, and iron accumulation in the basal ganglia are examples of normal age-related brain changes that can be seen on structural brain imaging. ${ }^{2}$ These changes, which are associated with cognitive decline, most often affect the domains of attention, memory, and executive function. ${ }^{3,4} \mathrm{AD}$ is accompanied by atrophy in the bilateral medial temporal, parietal, and frontal lobes. ${ }^{2}$ These changes are associated with serious cognitive decline and a number of neuropsychiatric symptoms.

However, depending on the disease progression, neurodegenerative manifestations, such as cerebral atrophy, are detected late in course of AD. Though CSF amyloid- $\beta(A \beta)$ 1-42 levels may begin decreasing 25 years before the onset of symptoms and brain amyloid deposition occurs in preclinical stage of $\mathrm{AD},{ }^{5}$ the gap between early amyloid detection and structural brain changes is wide. ${ }^{6}$ Early detection of $\mathrm{AD}$ is a key priority in dementia services and research. Therefore, additional biomarkers in the pathophysiological cascade of $\mathrm{AD}$ are needed, such as functional brain changes. Functional 
changes in the brain may be an indirect indicator of transsynaptic activity and they usually appear prior to structural changes in $\mathrm{AD}^{7}{ }^{7}$ Resting-state functional magnetic resonance imaging (RS-fMRI) has recently been highlighted as a new technique for interrogating intrinsic functional connectivity networks (ICN) without an experimentally determined context. ${ }^{8}$ Previous studies have shown that ICN changes are sensitive to functional brain changes related to Alzheimer's disease pathology across the clinical spectrum. ${ }^{9}$

Herein, we present a review of the current research on changes in functional connectivity, as measured by RS-fMRI. We compare researcher's outcomes from studies that examine normal healthy aging, patients with MCI, and AD.

\section{RESTING-STATE FMRI BASIC PRINCIPLES AND CORE LARGE-SCALE NETWORKS}

Recently, fMRI has largely replaced nuclear medicine techniques because it is non-invasive and does not require use of radioactive contrast media, instead, fMRI uses blood-oxygenlevel-dependent (BOLD) contrast. ${ }^{2}$ In BOLD fMRI, the difference in magnetic susceptibility between oxygenated and deoxygenated blood serves as an intrinsic contrast medium through which to compare conditions.

Initially, fMRI was developed to measure two conditions (e.g., task or no task) using an activation paradigm, so-called task-based fMRI. ${ }^{6}$ In recent years, there has been an increased interest in applying the technique to at-rest conditions, termed RS-fMRI. ${ }^{10}$ RS-fMRI focuses on spontaneous low-frequency fluctuations $(<0.1 \mathrm{~Hz})$ in the BOLD signal. ${ }^{10}$ This has led to the description of 10-20 RS networks, the most commonly observed of which are: the default mode network (DMN), the salience network (SN), the central executive network (CEN), the visual processing network, the sensorymotor network, the dorsal attention network, and auditory network, to name a few.

In many task-based fMRI studies, reversed contrasts revealed consistent deactivation of specific regions, ${ }^{11}$ including the medial prefrontal cortex ( $\mathrm{mPFC}$ ), the posterior cingulate cortex (PCC), the precuneus, the anterior cingulate cortex (ACC), the parietal cortex, and, in a minority of studies, the hippocampus. ${ }^{12}$ The default mode network (DMN) is so named because it is the most active network in the absence of a task. ${ }^{13}$ Among the majority of RS-fMRI studies, the DMN gained particular focus because alterations to its functional connectivity were observed in subjects who had $\mathrm{AD}$, who had $\mathrm{MCI}$, or who were at high risk for $\mathrm{AD}$. The $\mathrm{DMN}$ is known to be involved in the retrieval of autobiographical episodic memory as well as self-referential mental processing. ${ }^{14,15}$

Besides the DMN, other stable ICNs, such as the SN and the
CEN, have been known to play crucial roles in cognitive processing. ${ }^{16}$ The CEN includes the dorsolateral prefrontal cortex (DLPFC) and the posterior parietal cortex (PPC). ${ }^{17}$ It plays a key role in active maintenance and manipulation of information in working memory, and in judgment and decision-making in the context of goal-directed behavior. ${ }^{18-20}$ Additionally, the SN mainly consists of the dorsal ACC and the frontoinsular cortices (FIC). It serves to identify the most relevant stimuli for guiding behavior in response to several internal and external stimuli. ${ }^{21,22}$ The right FIC is a core node of the SN and plays a critical role in switching between the CEN and the $\mathrm{DMN},{ }^{23}$ which are known to interact competitively during cognitive information processing. ${ }^{24,25}$ Once a salient stimulus is detected, the FIC initiates the appropriate transient control signals to engage the CEN to mediate attention, working memory and other higher-order cognitive processes, all while disengaging the DMN. ${ }^{22}$ This switching mechanism helps to focus attention on external stimuli and eventually allows the stimuli to take on added significance or saliency. ${ }^{22}$

In this review, we focus on the DMN, SN, and CEN to describe RS-fMRI results from three groups: normal healthy aging, MCI and $\mathrm{AD}$.

\section{ANALYTIC TECHNIQUES}

There are three main analytic techniques used to assess functional connectivity: the seed-based approach, independent components analysis (ICA), and graph theory.

In the seed-based approach, the researcher selects a "seed" of interest (region of interest, ROI) and extracts the time course of activation at that ROI. This time course is then tested for correlation with the time courses of the rest of the voxels of the brain to search for matches. ${ }^{26}$ Brain regions that are positively correlated with each other are thought to belong to functionally coupled networks. ${ }^{27}$

In contrast, in ICA, the researcher does not need to select a reference region. Instead, the entire set of four-dimensional fMRI data can be decomposed into time courses and associated spatial maps that describe the temporal and spatial characteristics of the data components. ${ }^{28}$ ICA seeks underlying components of activation that are statistically independent of each other. Both seed-based and ICA approaches tend to reveal the same networks. However, seed-based analyses are sometimes preferred because they can make group comparisons easier. ${ }^{27}$

Studies using graph theory with RS-fMRI are emerging. In graph theory, ROIs can be represented as nodes. ${ }^{10}$ Nodes define anatomically homogeneous brain regions or individual voxels. ${ }^{29}$ The fMRI data is spatially parcellated according to a structural map of the brain, and correlations are computed between activations in all region pairs. ${ }^{27}$ Typical measure- 
ments taken under graph theory include average path length and global connectedness, which is the average length of the shortest connections between all pairs of nodes. ${ }^{30}$ Another measure of interest is the clustering coefficient, which is related to the connectedness of neighboring nodes and reflects the presence of small world structures. ${ }^{30}$

\section{RESTING-STATE FUNCTIONAL MRI ALTERATIONS IN THE NORMAL HEALTHY AGING}

RS-fMRI allows for the investigation of the neural bases of age-related cognitive changes, such as attention, information processing, and working memory. ${ }^{31,32}$ To date, the DMN has been the main focus of aging-related RS-fMRI research. Many studies reported that this network's activity during rest is necessary for memory consolidation. ${ }^{26}$ Additionally, amyloid deposition associated with $\mathrm{AD}$ is seen initially and most prominently in DMN hubs. ${ }^{12,33,34}$

Most of the RS-fMRI studies that focused on the DMN showed that aging are associated with decreased connectivity within this system. After assessing functional connectivity within the network, the seed-based and ICA studies produced similar results. Several studies reported reduced functional connectivity between anterior and posterior components of the DMN with advanced age. ${ }^{35,36} \mathrm{Wu}$ et al..$^{37}$ found greater integrity and greater preparation of DMN components in younger subjects compared to elderly subjects. The reduced RS-fMRI activity seen in the DMN in normal healthy aging subjects was correlated with executive dysfunction and decreased processing speed. ${ }^{2}$ Graph analysis studies further showed that decreased global connectivity and decreased nodal efficiency in several major brain hubs became more common during the aging process. ${ }^{38}$ Studies that focused on the modularity of functional networks reported that modularity decreased with age, indicating changes in size, composition, and the topological roles of the networks. ${ }^{39}$

Age-related resting-state functional connectivity changes have been reported not only in the DMN but also in the SN and the CEN. Because there are fewer studies of the SN and the CEN, age-related resting-state functional connectivity changes in these regions are not yet well understood. Onoda et al. $^{40}$ reported that connectivity within the $\mathrm{SN}$ decreased and certain internetwork connectivities (e.g., SN to auditory network, DMN to visual processing network, etc.) also decreased with aging. He et al. ${ }^{41}$ found age-related decreases in the positive functional connectivities between the right FIC in $\mathrm{SN}$ and the CEN and in the negative functional connectivities between the right FIC and the DMN, with and without atrophy correction. In a later study, $\mathrm{He}$ et al..$^{42}$ also found that functional impairments of the SN may occur as early as in normal aging.

Several hypotheses have been formulated to explain the functional connectivity deficits associated with non-pathological brain aging. Age-related changes in resting state functional connectivity are thought to reflect declines in structural connectivity, especially in white matter integrity, dopaminergic deficit, and amyloid deposition in the brain. ${ }^{43}$

\section{RESTING-STATE FMRI ALTERATIONS IN PATIENTS WITH MCI AND AD}

Most seed-based and ICA studies indicate that there is decreased connectivity in the DMN and other networks in subjects with MCI and AD. The majority of RS-fMRI studies found reduced DMN connectivity across widespread regions in the frontal, parietal, temporal and occipital regions of $\mathrm{AD}$ patients. ${ }^{44,45}$ Using graph analysis, major hubs in the brain seem to be affected by MCI and AD. Supkar et al. ${ }^{46}$ observed the loss of small-world organization in the functional brain network, characterized by a significantly lower clustering coefficient, which is indicative of disrupted local connectivity. Brier et al. ${ }^{47}$ also found that the clustering coefficient and modularity were lower in $\mathrm{AD}$ patients. In the same study, cognitively normal participants who harbor $\mathrm{AD}$ pathologies also showed reduced functional connectivity, demonstrating brain changes similar to, but smaller than, those seen in $\mathrm{AD} .^{47}$

However, results regarding aberrant functions in the DMN from MCI patients were different in patterns from those of AD patients. Damoiseaux et al. ${ }^{9}$ reported decreased connectivity in the posterior DMN alongside increased connectivity in the ventral and anterior DMN using ICA. Also using ICA, Qi et al. ${ }^{48}$ found that MCI patients showed a trend toward reduced hippocampus activity compared to healthy elderly. They also found that reduced functional activity in the bilateral precuneus/posterior cingulate cortex, the right inferior parietal lobule, and the left fusiform gyrus indicated network deficiency, while increased activity in the left prefrontal cortex, the inferior parietal lobule, and the middle temporal gyrus suggested network compensation in MCI patients. Using a seed-based approach, the most recent study of MCI patients reported increased DMN connectivity between the medial prefrontal regions and the posterior cingulate and between the posterior cingulated, the parahippocampus and the anterior hippocampus. ${ }^{49}$ These results suggest that the DMN functions aberrantly in MCI patients, which might be due to neuronal loss in the initial phase of neurodegeneration. Increased functional connectivity between the PCC, the parahippocampus, and the anterior hippocampus in MCI patients may reflect a maladaptive mechanism. ${ }^{49}$ 
Decreased DMN connectivity was even found in unaffected carriers of familial $\mathrm{AD}$ who have pathogenic presenilin-1 (PSEN1), presenilin-2 (PSEN2), or amyloid precursor protein (APP) mutations, ${ }^{50}$ as well as among subjects with cognitive complaints, but normal neuropsychological performance. ${ }^{51}$ Also, APOE $\varepsilon 4$ carriers showed decreased connectivity of the posterior DMN and relatively increased connectivity of the SN. ${ }^{52}$ However, PSEN1-mutation carriers who are between 9 to 17 years old demonstrated increased functional connectivity between the posterior cingulate cortex and the medial temporal lobe regions, as well as high gray-matter volume in their temporal regions. ${ }^{53}$

Table 1. Schematic summary of the main group differences in RS-fMRI obtained using both the seed-based and independent components analysis (ICA) methods

\begin{tabular}{|c|c|c|c|c|}
\hline \multirow{2}{*}{ Reference (year) } & \multicolumn{3}{|l|}{ Intra-network } & \multirow{2}{*}{ Inter-network } \\
\hline & $\mathrm{DMN}$ & $\mathrm{SN}$ & CEN & \\
\hline \multicolumn{5}{|l|}{ Healthy aging } \\
\hline Andrews-Hanna et al. $(2007)^{35}$ & $\downarrow$ & NA & NA & NA \\
\hline Damoiseaux et al. $(2008)^{36}$ & $\downarrow$ & NA & NA & NA \\
\hline Wu et al. $(2011)^{37}$ & $\downarrow$ & NA & NA & NA \\
\hline \multirow[t]{2}{*}{ Onoda et al. $(2012)^{40}$} & NA & $\downarrow$ & NA & $\downarrow$ SN-auditory \\
\hline & & & & $\downarrow D M N$-auditory \\
\hline \multirow[t]{2}{*}{ He et al. $(2014)^{42}$} & NA & $\downarrow$ & NA & $\downarrow$ SN-CEN \\
\hline & & & & $\downarrow$ SN-DMN \\
\hline \multicolumn{5}{|c|}{ Amyloid (+) cognitively normal subjects } \\
\hline Hedden et al. $(2009)^{33}$ & $\downarrow$ & NA & NA & NA \\
\hline Mormino et al. $(2011)^{34}$ & $\downarrow$ & NA & NA & NA \\
\hline Sheline et al. $(2010)^{54}$ & $\downarrow$ & NA & NA & NA \\
\hline Lim et al. $(2014)^{56}$ & $\uparrow$ & - & $\downarrow$ & $\downarrow$ DMN-CEN \\
\hline \multicolumn{5}{|l|}{ Mild cognitive impairment } \\
\hline \multirow[t]{2}{*}{ Damoiseaux et al. $(2012)^{9}$} & $\downarrow$ Posterior & NA & NA & NA \\
\hline & $\uparrow$ Ventral, anterior & & & \\
\hline \multirow[t]{2}{*}{ Qi et al. $(2010)^{48}$} & $\downarrow$ Precuneus/PCC, Rt. Inf. parietal, Lt. fusiform & NA & NA & NA \\
\hline & $\uparrow$ Lt. PFC, Inf. parietal, middle temporal & & & \\
\hline Gardini et al. $(2015)^{49}$ & $\uparrow$ & NA & NA & NA \\
\hline Sorg et al. $(2007)^{59}$ & $\downarrow$ & NA & NA & NA \\
\hline Agosta et al. $(2012)^{60}$ & $\downarrow$ & NA & NA & NA \\
\hline \multirow[t]{2}{*}{ He et al. $(2014)^{42}$} & NA & NA & NA & $\downarrow$ SN-CEN \\
\hline & & & & $\downarrow \mathrm{SN}-\mathrm{DMN}$ \\
\hline Brier et al. $(2012)^{44}$ & $\downarrow$ & $\downarrow$ & NA & NA \\
\hline Wang et al. $(2013)^{51}$ & $\downarrow$ & NA & NA & NA \\
\hline Bai et al. $(2008)^{62}$ & $\downarrow$ & NA & NA & NA \\
\hline \multicolumn{5}{|l|}{ Alzheimer's disease } \\
\hline Balachandar et al. $(2015)^{7}$ & $\downarrow$ & NA & $\uparrow$ & NA \\
\hline Brier et al. $(2012)^{44}$ & $\downarrow$ & $\downarrow$ & NA & NA \\
\hline Zhang et al. $(2009)^{45}$ & $\downarrow$ & NA & $\uparrow$ & NA \\
\hline Agosta et al. $(2012)^{60}$ & $\downarrow$ & $\uparrow$ & $\uparrow$ & NA \\
\hline Zhou et al. $(2010)^{61}$ & $\downarrow$ & $\uparrow$ & NA & NA \\
\hline \multirow[t]{2}{*}{ He et al. $(2014)^{42}$} & NA & $\downarrow$ & NA & $\downarrow$ SN-CEN \\
\hline & & & & $\downarrow$ SN-DMN \\
\hline
\end{tabular}

$\overline{\mathrm{DMN}}$ : default mode network, SN: salience network, CEN: central executive network, $\downarrow$ : decreased functional connectivity, $\uparrow$ : increased functional connectivity, - : not different, NA: not applicable, PCC: posterior cingulate cortex, PFC: prefrontal cortex. RS-fMRI: Resting-state functional magnetic resonance imaging 
There are several studies that look into the association between amyloid- $\beta$ deposition and functional brain connectivity. Several previous studies reported that reduced functional connectivity of the DMN can be found in cognitively normal subjects with brain amyloid burden. ${ }^{33,34,54}$ Using graph analysis, a decreased number of hubs and reduced connectivity within the DMN were found both $\mathrm{MCI}$ and $\mathrm{AD}$, as well as in healthy elderly with amyloid- $\beta$ deposition on positron emission tomography (PET) images. ${ }^{33,55}$ However, Lim et al. ${ }^{56}$ reported that the functional connectivity of the DMN among older adults with normal cognition was greater, while the SN was not different, and the CEN was lower in among the Pittsburgh compound B-positive group, compared to the Pittsburgh compound B-negative group. The authors explained that the discrepancy in DMN result might be attributed to the "acceleration" and "brain reserve" hypotheses. The acceleration hypothesis predicts that the initiation of amyloid- $\beta$ deposition by independent events instigates a milieu of higher functional connectivity that accelerates this deposition, which eventually leads to functional disconnection or metabolic deterioration in subjects with amyloid burden..$^{57,58}$ The brain reserve hypothesis states that higher brain metabolism and functional connectivity represent stable traits that impart the capacity to withstand amyloid- $\beta$ deposition and maintain normal cognition. ${ }^{57}$

Results in the SN and CEN appear to be less consistent. Sorg et al. ${ }^{59}$ found reduced connectivity in the DMN and central executive network (CEN) hubs of MCI patients. Agosta et al. ${ }^{60}$ reported decreased connectivity in the DMN among MCI and $\mathrm{AD}$ patients, as well as increased mean connectivity in the CEN among $\mathrm{AD}$ patients. Balachandar et al. ${ }^{7}$ reported decreased connectivity in the DMN and increased connectivity in CEN in mild $\mathrm{AD}$ patients. Zhou et al. ${ }^{61}$ reported increased functional connectivity in the $\mathrm{SN}$ of $\mathrm{AD}$ patients. However, He et al. ${ }^{42}$ found that both the structural and functional organizations of the $\mathrm{SN}$ are impaired in $\mathrm{MCI}$ and $\mathrm{AD}$ patients.

The summary of the main group differences in RS-fMRI obtained using both the seed-based and independent components analysis (ICA) methods is provided in Table 1 .

\section{CONCLUSIONS}

According to a number of empirical studies, subjects who are aging healthfully, those who have MCI and those who have $\mathrm{AD}$ all show changes in functional connectivity in three large-scale networks-the DMN, $\mathrm{SN}$, and CEN-based on RSfMRI results. These findings help build an understanding of the neural substrates in the aging brain and during pathological degenerative processes.

Previous studies have shown that the ICN changes that are observable in RS-fMRI are sensitive to the functional brain changes that are often seen across the spectrum of $\mathrm{AD}$ pathology. ${ }^{9}$ The ICN changes seen on RS-fMRI are potentially sensitive, non-invasive biomarkers of AD. Among the ICNs, the DMN, SN and CEN have turned out to be particularly important for maintaining higher cognitive function; hence, the use of the term "core" neurocognitive networks. In particular, the DMN has been the most investigated resting-state network. And most RS-fMRI studies report reduced DMN connectivity in normally aging, $\mathrm{MCI}$ and $\mathrm{AD}$ subjects. However, reduced DMN connectivity has also been reported to be associated with a variety of other diseases, such as Parkinson's disease and multiple sclerosis, and is by no means specific to AD. ${ }^{6}$ Besides, DMN, SN and CEN abnormalities are widespread in psychiatric, as well as in neurological disorders. ${ }^{16}$ Different studies have revealed that there are both consistencies and discrepancies in ICN changes among normally aging, $\mathrm{MCI}$ and $\mathrm{AD}$ subjects, possibly due to methodological differences and/or clinical heterogeneity among subjects. ${ }^{62}$ These limitations have recently been overcome and it is expected that clinical applications of RS-fMRI in normal aging, $\mathrm{MCI}$ and $\mathrm{AD}$ will continue to increase.

\section{REFERENCES}

1. Williams KN, Kemper S. Interventions to reduce cognitive decline in aging. J Psychosoc Nurs Ment Health Serv 2010;48:42-51.

2. Barkhof F, Fox NC, Bastos-Leite AJ, Scheltens P. Neuroimaging in Dementia. New York: Springer-Verlag Berlin Heidelberg; 2011.

3. Hedden T, Gabrieli JD. Insights into the ageing mind: a view from cognitive neuroscience. Nat Rev Neurosci 2004;5:87-96.

4. Whalley LJ, Deary IJ, Appleton CL, Starr JM. Cognitive reserve and the neurobiology of cognitive aging. Ageing Res Rev 2004;3:369-382.

5. Bateman RJ, Xiong C, Benzinger TL, Fagan AM, Goate A, Fox NC, et al. Clinical and biomarker changes in dominantly inherited Alzheimer's disease. N Engl J Med 2012;367:795-804.

6. Barkhof F, Haller S, Rombouts SA. Resting-state functional MR imaging: a new window to the brain. Radiology 2014;272:29-49.

7. Balachandar R, John JP, Saini J, Kumar KJ, Joshi H, Sadanand S, et al. A study of structural and functional connectivity in early Alzheimer's disease using rest fMRI and diffusion tensor imaging. Int J Geriatr Psychiatry 2015;30:497-504.

8. Vemuri P, Jones DT, Jack CR Jr. Resting state functional MRI in Alzheimer's disease. Alzheimers Res Ther 2012;4:2.

9. Damoiseaux JS, Prater KE, Miller BL, Greicius MD. Functional connectivity tracks clinical deterioration in Alzheimer's disease. Neurobiol Aging 2012;33:828 e819-830.

10. Lee MH, Smyser CD, Shimony JS. Resting-state fMRI: a review of methods and clinical applications. AJNR Am J Neuroradiol 2013;34: 1866-1872.

11. Gusnard DA, Raichle ME, Raichle ME. Searching for a baseline: functional imaging and the resting human brain. Nat Rev Neurosci 2001; 2:685-694.

12. Buckner RL, Andrews-Hanna JR, Schacter DL. The brain's default network: anatomy, function, and relevance to disease. Ann N Y Acad Sci 2008;1124:1-38.

13. Raichle ME, MacLeod AM, Snyder AZ, Powers WJ, Gusnard DA, Shulman GL. A default mode of brain function. Proc Natl Acad Sci U 
S A 2001;98:676-682.

14. Spreng RN, Mar RA, Kim AS. The common neural basis of autobiographical memory, prospection, navigation, theory of mind, and the default mode: a quantitative meta-analysis. J Cogn Neurosci 2009;21: 489-510.

15. Whitfield-Gabrieli S, Moran JM, Nieto-Castanon A, Triantafyllou C, Saxe R, Gabrieli JD. Associations and dissociations between default and self-reference networks in the human brain. Neuroimage 2011;55: 225-232.

16. Menon V. Large-scale brain networks and psychopathology: a unifying triple network model. Trends Cogn Sci 2011;15:483-506.

17. Damoiseaux JS, Rombouts SA, Barkhof F, Scheltens P, Stam CJ, Smith $\mathrm{SM}$, et al. Consistent resting-state networks across healthy subjects. Proc Natl Acad Sci U S A 2006;103:13848-13853.

18. Petrides M. Lateral prefrontal cortex: architectonic and functional organization. Philos Trans R Soc Lond B Biol Sci 2005;360:781-795.

19. Muller NG, Knight RT. The functional neuroanatomy of working memory: contributions of human brain lesion studies. Neuroscience 2006;139:51-58.

20. Koechlin E, Summerfield C. An information theoretical approach to prefrontal executive function. Trends Cogn Sci 2007;11:229-235.

21. Seeley WW, Menon V, Schatzberg AF, Keller J, Glover GH, Kenna H, et al. Dissociable intrinsic connectivity networks for salience processing and executive control. J Neurosci 2007;27:2349-2356.

22. Menon V, Uddin LQ. Saliency, switching, attention and control: a network model of insula function. Brain Struct Funct 2010;214:655-667.

23. Sridharan D, Levitin DJ, Menon V. A critical role for the right frontoinsular cortex in switching between central-executive and defaultmode networks. Proc Natl Acad Sci U S A 2008;105:12569-12574.

24. Greicius MD, Krasnow B, Reiss AL, Menon V. Functional connectivity in the resting brain: a network analysis of the default mode hypothesis. Proc Natl Acad Sci U S A 2003;100:253-258.

25. Fox MD, Snyder AZ, Vincent JL, Corbetta M, Van Essen DC, Raichle ME. The human brain is intrinsically organized into dynamic, anticorrelated functional networks. Proc Natl Acad Sci U S A 2005;102:96739678.

26. Fox MD, Raichle ME. Spontaneous fluctuations in brain activity observed with functional magnetic resonance imaging. Nat Rev Neurosci 2007;8:700-711.

27. Dennis EL, Thompson PM. Functional brain connectivity using fMRI in aging and Alzheimer's disease. Neuropsychol Rev 2014;24:49-62.

28. Beckmann CF, DeLuca M, Devlin JT, Smith SM. Investigations into resting-state connectivity using independent component analysis. Philos Trans R Soc Lond B Biol Sci 2005;360:1001-1013.

29. Stam CJ, de Haan W, Daffertshofer A, Jones BF, Manshanden I, van Cappellen van Walsum AM, et al. Graph theoretical analysis of magnetoencephalographic functional connectivity in Alzheimer's disease. Brain 2009;132:213-224.

30. van den Heuvel MP, Stam CJ, Boersma M, Hulshoff Pol HE. Smallworld and scale-free organization of voxel-based resting-state functional connectivity in the human brain. Neuroimage 2008;43:528-539.

31. Park DC, Smith AD, Lautenschlager G, Earles JL, Frieske D, Zwahr M, et al. Mediators of long-term memory performance across the life span. Psychol Aging 1996;11:621-637.

32. Salthouse TA, Ferrer-Caja E. What needs to be explained to account for age-related effects on multiple cognitive variables? Psychol Aging 2003;18:91-110.

33. Hedden T, Van Dijk KR, Becker JA, Mehta A, Sperling RA, Johnson KA, et al. Disruption of functional connectivity in clinically normal older adults harboring amyloid burden. J Neurosci 2009;29:12686-12694.

34. Mormino EC, Smiljic A, Hayenga AO, Onami SH, Greicius MD, Rabinovici GD, et al. Relationships between $\beta$-amyloid and functional connectivity in different components of the default mode network in aging. Cereb Cortex 2011;21:2399-2407.

35. Andrews-Hanna JR, Snyder AZ, Vincent JL, Lustig C, Head D, Raichle
ME, et al. Disruption of large-scale brain systems in advanced aging. Neuron 2007;56:924-935.

36. Damoiseaux JS, Beckmann CF, Arigita EJ, Barkhof F, Scheltens P, Stam CJ, et al. Reduced resting-state brain activity in the "default network" in normal aging. Cereb Cortex 2008;18:1856-1864.

37. Wu JT, Wu HZ, Yan CG, Chen WX, Zhang HY, He Y, et al. Aging-related changes in the default mode network and its anti-correlated networks: a resting-state fMRI study. Neurosci Lett 2011;504:62-67.

38. Achard S, Bullmore E. Efficiency and cost of economical brain functional networks. PLoS Comput Biol 2007;3:e17.

39. Meunier D, Achard S, Morcom A, Bullmore E. Age-related changes in modular organization of human brain functional networks. Neuroimage 2009;44:715-723.

40. Onoda K, Ishihara M, Yamaguchi S. Decreased functional connectivity by aging is associated with cognitive decline. J Cogn Neurosci 2012; 24:2186-2198.

41. He X, Qin W, Liu Y, Zhang X, Duan Y, Song J, et al. Age-related decrease in functional connectivity of the right fronto-insular cortex with the central executive and default-mode networks in adults from young to middle age. Neurosci Lett 2013;544:74-79.

42. He X, Qin W, Liu Y, Zhang X, Duan Y, Song J, et al. Abnormal salience network in normal aging and in amnestic mild cognitive impairment and Alzheimer's disease. Hum Brain Mapp 2014;35:3446-3464.

43. Ferreira LK, Busatto GF. Resting-state functional connectivity in normal brain aging. Neurosci Biobehav Rev 2013;37:384-400.

44. Brier MR, Thomas JB, Snyder AZ, Benzinger TL, Zhang D, Raichle $\mathrm{ME}$, et al. Loss of intranetwork and internetwork resting state functional connections with Alzheimer's disease progression. J Neurosci 2012;32:8890-8899.

45. Zhang HY, Wang SJ, Xing J, Liu B, Ma ZL, Yang M, et al. Detection of PCC functional connectivity characteristics in resting-state fMRI in mild Alzheimer's disease. Behav Brain Res 2009;197:103-108.

46. Supekar K, Menon V, Rubin D, Musen M, Greicius MD. Network analysis of intrinsic functional brain connectivity in Alzheimer's disease. PLoS Comput Biol 2008;4:e1000100.

47. Brier MR, Thomas JB, Fagan AM, Hassenstab J, Holtzman DM, Benzinger TL, et al. Functional connectivity and graph theory in preclinical Alzheimer's disease. Neurobiol Aging 2014;35:757-768.

48. Qi Z, Wu X, Wang Z, Zhang N, Dong H, Yao L, et al. Impairment and compensation coexist in amnestic MCI default mode network. Neuroimage 2010;50:48-55.

49. Gardini S, Venneri A, Sambataro F, Cuetos F, Fasano F, Marchi M, et al. Increased functional connectivity in the default mode network in mild cognitive impairment: a maladaptive compensatory mechanism associated with poor semantic memory performance. J Alzheimers Dis 2015;45:457-470.

50. Chhatwal JP, Schultz AP, Johnson K, Benzinger TL, Jack C Jr, Ances $\mathrm{BM}$, et al. Impaired default network functional connectivity in autosomal dominant Alzheimer disease. Neurology 2013;81:736-744.

51. Wang Y, Risacher SL, West JD, McDonald BC, Magee TR, Farlow MR, et al. Altered default mode network connectivity in older adults with cognitive complaints and amnestic mild cognitive impairment. J Alzheimers Dis 2013;35:751-760.

52. Machulda MM, Jones DT, Vemuri P, McDade E, Avula R, Przybelski S, et al. Effect of APOE epsilon4 status on intrinsic network connectivity in cognitively normal elderly subjects. Arch Neurol 2011;68:1131-1136.

53. Quiroz YT, Schultz AP, Chen K, Protas HD, Brickhouse M, Fleisher AS, et al. Brain imaging and blood biomarker abnormalities in children with autosomal dominant Alzheimer disease: a cross-sectional study. JAMA Neurol 2015;72:912-919.

54. Sheline YI, Raichle ME, Snyder AZ, Morris JC, Head D, Wang S, et al. Amyloid plaques disrupt resting state default mode network connectivity in cognitively normal elderly. Biol Psychiatry 2010;67:584-587.

55. Sheline YI, Raichle ME. Resting state functional connectivity in preclinical Alzheimer's disease. Biol Psychiatry 2013;74:340-347. 
56. Lim HK, Nebes R, Snitz B, Cohen A, Mathis C, Price J, et al. Regional amyloid burden and intrinsic connectivity networks in cognitively normal elderly subjects. Brain 2014;137:3327-3338.

57. Cohen AD, Price JC, Weissfeld LA, James J, Rosario BL, Bi W, et al. Basal cerebral metabolism may modulate the cognitive effects of Abeta in mild cognitive impairment: an example of brain reserve. J Neurosci 2009;29:14770-14778.

58. Johnson SC, Christian BT, Okonkwo OC, Oh JM, Harding S, Xu G, et al. Amyloid burden and neural function in people at risk for Alzheimer's disease. Neurobiol Aging 2014;35:576-584.

59. Sorg C, Riedl V, Muhlau M, Calhoun VD, Eichele T, Laer L, et al. Selective changes of resting-state networks in individuals at risk for $\mathrm{Al}$ - zheimer's disease. Proc Natl Acad Sci U S A 2007;104:18760-18765.

60. Agosta F, Pievani M, Geroldi C, Copetti M, Frisoni GB, Filippi M. Resting state fMRI in Alzheimer's disease: beyond the default mode network. Neurobiol Aging 2012;33:1564-1578.

61. Zhou J, Greicius MD, Gennatas ED, Growdon ME, Jang JY, Rabinovici GD, et al. Divergent network connectivity changes in behavioural variant frontotemporal dementia and Alzheimer's disease. Brain 2010;133: 1352-1367.

62. Bai F, Zhang Z, Yu H, Shi Y, Yuan Y, Zhu W, et al. Default-mode network activity distinguishes amnestic type mild cognitive impairment from healthy aging: a combined structural and resting-state functional MRI study. Neurosci Lett 2008;438:111-115. 